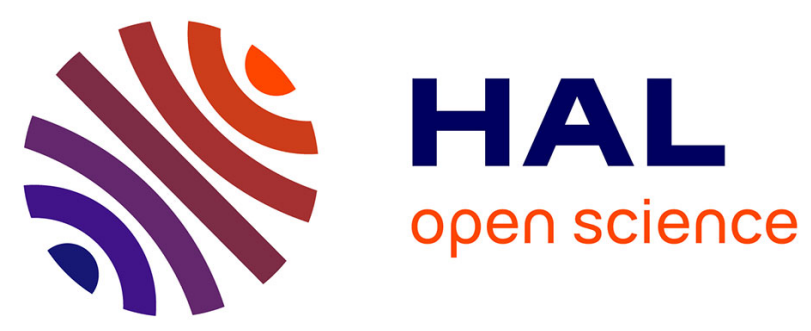

\title{
La mobilisation des bois en provenance des forêts bénéficiant du régime forestier en 1994 et les prix obtenus aux grandes ventes de l'automne 1994 - Calendrier des grandes ventes d'automne 1995
}

- [.]office National Des Forets Paris

\section{To cite this version:}

- [.]office National Des Forets Paris. La mobilisation des bois en provenance des forêts bénéficiant du régime forestier en 1994 et les prix obtenus aux grandes ventes de l'automne 1994 - Calendrier des grandes ventes d'automne 1995. Revue forestière française, 1995, 67 (4), pp.309-328. $10.4267 / 2042 / 26662$. hal-03535421

\author{
HAL Id: hal-03535421 \\ https://hal.science/hal-03535421
}

Submitted on 19 Jan 2022

HAL is a multi-disciplinary open access archive for the deposit and dissemination of scientific research documents, whether they are published or not. The documents may come from teaching and research institutions in France or abroad, or from public or private research centers.
L'archive ouverte pluridisciplinaire HAL, est destinée au dépôt et à la diffusion de documents scientifiques de niveau recherche, publiés ou non, émanant des établissements d'enseignement et de recherche français ou étrangers, des laboratoires publics ou privés. 


\title{
LA MOBILISATION DES BOIS EN PROVENANCE DES FORÊTS BÉNÉFICIANT DU RÉGIME FORESTIER EN 1994 ET LES PRIX OBTENUS AUX GRANDES VENTES DE L'AUTOMNE 1994
}

\author{
OFFICE NATIONAL DES FORÊTS
}

La reprise économique générale n'a pas manqué d'avoir une incidence sur le marché du bois en 1994.

Sur le plan des résineux, l'augmentation des mises en chantiers aux États-Unis conjuguée à un plafonnement de l'offre canadienne de bois a permis l'ouverture de nouveaux débouchés pour les bois scandinaves, entraînant parallèlement une revalorisation très sensible de leurs prix sur le marché international ( $+50 \%$ sur les résineux blancs et $+35 \%$ sur les bois rouges entre novembre 1992 , point le plus bas, et juillet 1994).

En France, la remontée des prix des sciages de résineux scandinaves à l'importation, à laquelle s'est ajoutée l'amélioration de l'activité du bâtiment a redonné une bouffée d'air aux sciages de pays.

De plus, les stocks des exploitants-scieurs étant très bas, les besoins en réapprovisionnement ont été pressants et ont relancé une certaine concurrence dans les ventes de bois tant sur pied que façonnés dès le deuxième trimestre de 1994.

Sur le plan des feuillus, la reprise économique en Europe se traduisait par un redémarrage des exportations françaises de Chêne et de Hêtre ainsi que de meubles et sièges en bois.

Enfin, le marché international de la pâte connaissait un redressement spectaculaire dont témoignait le dégonflement des stocks Norscan et la hausse continue des cours à compter du quatrième trimestre de 1993.

Moyennant quoi, les résultats des ventes d'automne de l'ONF ont fait apparaître des invendus très réduits et des cours en nette hausse pour la plupart des essences et des qualités de bois.

La mobilisation effective de bois en forêt publique s'est chiffrée globalement à près de 14,1 millions de mètres cubes, en augmentation de $5 \%$ sur 1993. Ce niveau est un niveau-record hors chablis exceptionnels de 1990. II a pu être atteint grâce à un phénomène de déstockage (les invendus ayant 
diminué de plus de moitié entre le 31 décembre 1993 et le 31 décembre 1994), mais aussi d'augmentation de l'offre de bois frais (bois mis en vente pour la première fois) en forêt des collectivités.

La récolte de bois a été mobilisée à $82 \%$ sur pied ( $79 \%$ en bloc et $3 \%$ à l'unité de produits) et $18 \%$ abattus et façonnés.

La part des bois commercialisés avec appel à la concurrence, soit en adjudication, soit en appel d'offres, s'élève à $73 \%$ et celle des bois vendus à l'amiable à $16 \%$ tandis que le reste $(11 \%)$ a été délivré aux collectivités propriétaires pour l'affouage ou l'autoconsommation.

\section{Graphique 1 MODES DE MISE EN MARCHÉ - ANNÉE 1994}

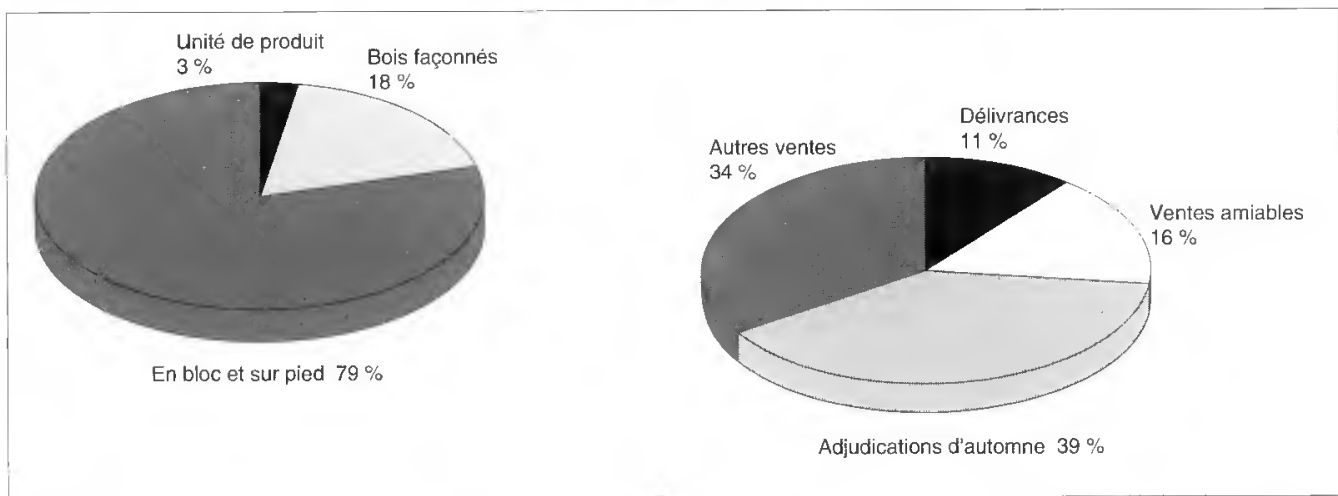

Tableau I

La mobilisation des bois en forêts soumises en 1994

\begin{tabular}{|c|c|c|c|c|c|}
\hline \multirow{2}{*}{ (En $\left.1000 \mathrm{~m}^{3}\right)$} & \multirow{2}{*}{$\begin{array}{c}\text { Forêt } \\
\text { domaniale }\end{array}$} & \multicolumn{2}{|c|}{ Forêt des collectivités } & \multirow{2}{*}{$\begin{array}{l}\text { Toutes forêts } \\
\text { soumises }\end{array}$} & \multirow{2}{*}{$\begin{array}{l}\text { Rappel forêts } \\
\text { soumises } 1993\end{array}$} \\
\hline & & Ventes & Délivrances & & \\
\hline 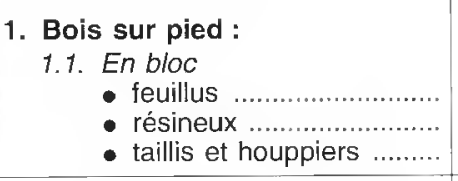 & $\begin{array}{l}2015 \\
1760 \\
1225 \\
\end{array}$ & $\begin{array}{r}1215 \\
2505 \\
970 \\
\end{array}$ & $\begin{array}{r}355 \\
55 \\
1000 \\
\end{array}$ & $\begin{array}{l}3585 \\
4320 \\
3195 \\
\end{array}$ & $\begin{array}{l}3490 \\
4075 \\
3035\end{array}$ \\
\hline 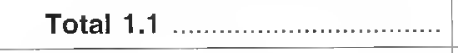 & 5000 & 4690 & 1410 & 11100 & 10600 \\
\hline $\begin{array}{l}\text { 1.2. À l'unité de produits } \\
\text { - feuillus .......................... } \\
\text { - résineux ...................... } \\
\text { - bois enstérés ou pesés }\end{array}$ & $\begin{array}{r}35 \\
50 \\
105\end{array}$ & $\begin{array}{r}5 \\
30 \\
205 \\
\end{array}$ & - & $\begin{array}{r}40 \\
80 \\
310 \\
\end{array}$ & $\begin{array}{r}30 \\
70 \\
260 \\
\end{array}$ \\
\hline 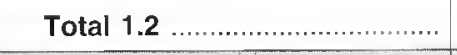 & 190 & 240 & 0 & 430 & 360 \\
\hline 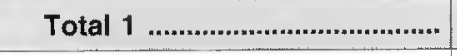 & 5190 & 4930 & 1410 & 11530 & 10960 \\
\hline 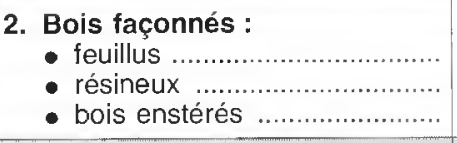 & $\begin{array}{r}390 \\
555 \\
95\end{array}$ & $\begin{array}{l}660 \\
605 \\
150\end{array}$ & - & $\begin{array}{r}1050 \\
1160 \\
355\end{array}$ & $\begin{array}{r}980 \\
1050 \\
390\end{array}$ \\
\hline Total $2 \ldots \ldots \ldots \ldots \ldots \ldots \ldots \ldots \ldots \ldots \ldots$ & 1040 & 1415 & 110 & 2565 & 2420 \\
\hline Total $1+2 \ldots \ldots \ldots \ldots \ldots \ldots \ldots$ & 6230 & 6345 & 1520 & 14095 & 13380 \\
\hline 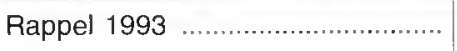 & 6200 & 5850 & 1330 & 13380 & \\
\hline
\end{tabular}




\section{LES RECETTES-BOIS}

La revalorisation des recettes-bois a été plus forte en forêt des collectivités qu'en forêt domaniale.

En effet, en forêt domaniale, elle est presque exclusivement liée à la hausse des cours $(+16 \%$ en moyenne) alors qu'en forêt des collectivités, elle repose à la fois sur la hausse des cours $(+14 \%$ en moyenne) et sur l'augmentation des volumes vendus (+ $8,5 \%)$.

Tableau II

Recettes bois *

\begin{tabular}{|c|c|c|c|c|c|c|}
\hline \multirow{2}{*}{ (En millions de F) } & \multicolumn{3}{|c|}{ Forêt domaniale } & \multicolumn{3}{|c|}{ Forêt des collectivités } \\
\hline & 1989 & 1993 & 1994 & 1989 & 1993 & 1994 \\
\hline 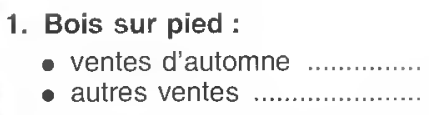 & $\begin{array}{l}951,0 \\
233,2\end{array}$ & $\begin{array}{l}639,0 \\
272,7\end{array}$ & $\begin{array}{l}816,9 \\
252,7\end{array}$ & $\begin{array}{l}622,9 \\
285,0\end{array}$ & $\begin{array}{l}471,0 \\
283,9\end{array}$ & $\begin{array}{l}650,3 \\
348,3\end{array}$ \\
\hline Total & 1184,2 & 911,7 & 1069,6 & 907,9 & 754,9 & 998,6 \\
\hline $\begin{array}{l}\text { 2. Bois façonnés : } \\
\text { - ventes }\end{array}$ & 327,4 & 309,2 & 350,4 & 514,9 & 527,2 & 583,8 \\
\hline Total ventes ...... & 1511,6 & 1220,9 & 1420,0 & 1422,8 & 1282,1 & 1582,4 \\
\hline $\begin{array}{l}\text { Estimation en valeur } \\
\text { des délivrances .......... }\end{array}$ & 0,3 & 2,7 & 3,1 & 100,3 & 63,8 & 62,5 \\
\hline
\end{tabular}

* n.c. recettes-bois DOM et menus produits ligneux.

\section{La répartition des recettes-bois par essence}

En 1994, les feuillus ont pesé pour près des deux-tiers dans les recettes domaniales de bois et pour un peu plus de la moitié dans celles des collectivités.

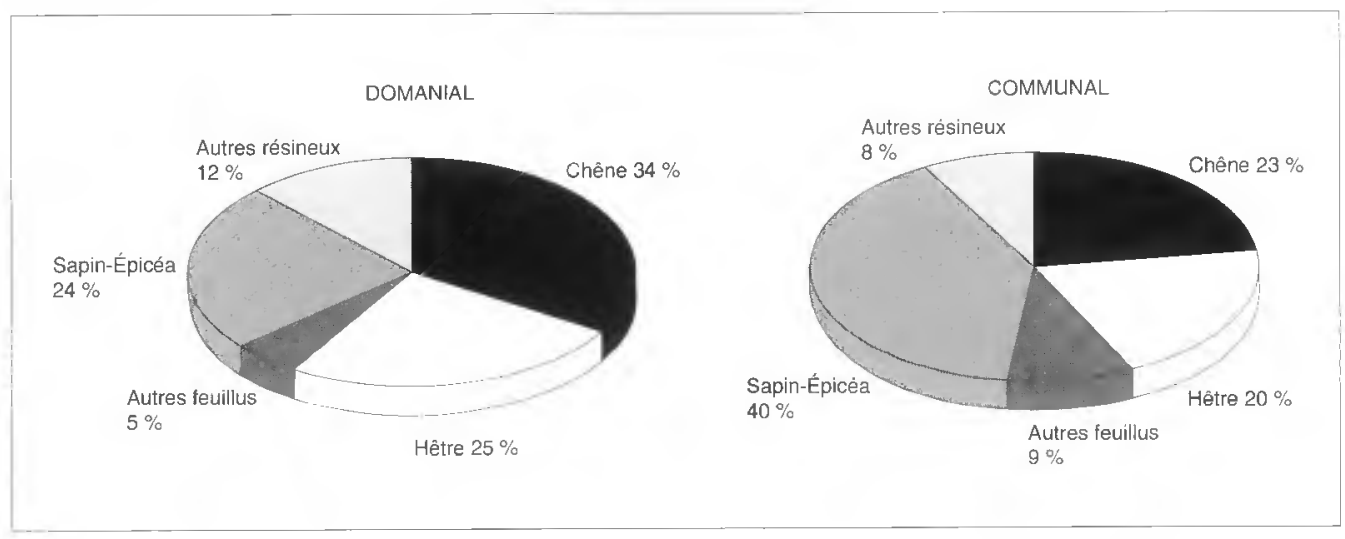




\section{OFFICE NATIONAL DES FORÊTS}

\section{LE CHÊNE}

Des quantités mobilisées importantes (près de $1800000 \mathrm{~m}^{3}$ volumes-grumes) et une revalorisation certaine des prix (+ $12 \%$ par rapport à 1993) témoignent de la reprise du marché du Chêne (cf. diagramme 1, ci-dessous).

Toutefois, cette revalorisation n'a pas suffi à effacer quatre années de baisse consécutive et permet à peine de rejoindre, en francs courants, le niveau de 1991 (cf. graphique 3, ci-dessous).

Cette revalorisation a été quasi générale et a touché toutes les qualités à peu près dans la même mesure. La baisse apparente du prix moyen dans quelques départements producteurs (Loir-et-Cher en particulier) ne reflète qu'une diminution de la qualité de l'échantillon offert (cf. tableaux III et IV, p. 313).

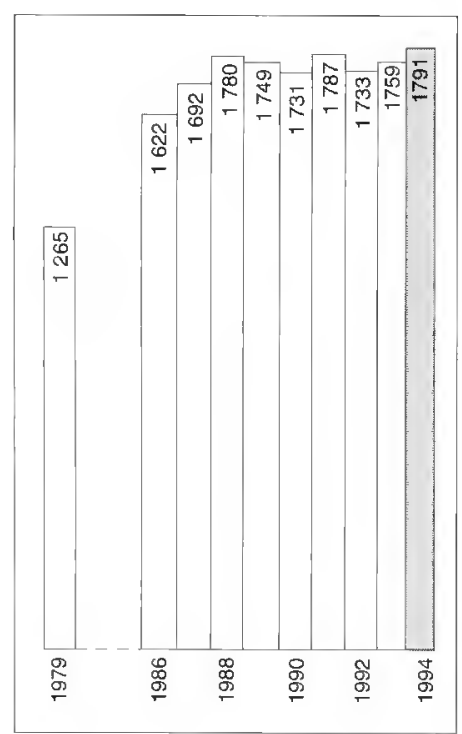

Diagramme 1

CHÊNE.

VOLUMES-GRUME MOBILISÉS

EN FORÊT SOUMISE (en $1000 \mathrm{~m}^{3}$ )

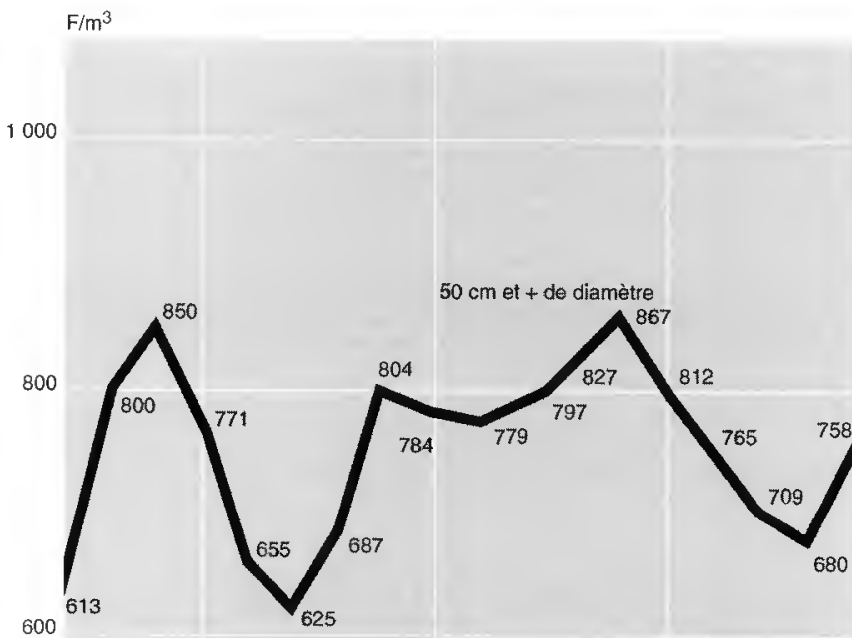

400

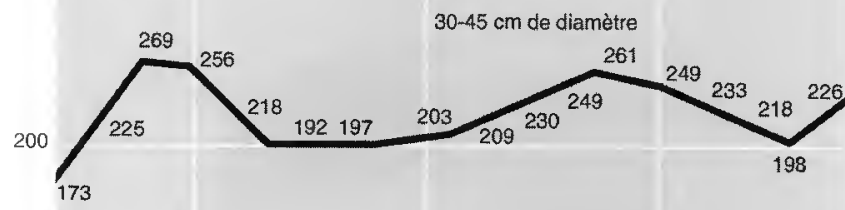

$25 \mathrm{~cm}$ et - de diamètre

Graphique 3

CHÊNE. ÉVOLUTION DES PRIX MOYENS AUX GRANDES VENTES
0 12 $\begin{array}{lllllllll}33 & 41 & 50 & 54 & 54 & 51 & 50 & 55 & 54\end{array}$ 
Chêne de $50 \mathrm{~cm}$ et plus de diamètre

Principaux départements producteurs - Grandes ventes 1994

\begin{tabular}{|c|c|c|c|c|}
\hline Départements & $\begin{array}{l}\text { Prix moyen } \\
1994\left(\mathrm{~F}^{3} \mathrm{~m}^{3}\right)\end{array}$ & $\begin{array}{c}\text { Variation } \\
1994 / 1993\end{array}$ & $\begin{array}{l}\text { Volume vendu } \\
\qquad\left(\mathrm{m}^{3}\right)\end{array}$ & $\begin{array}{l}\text { Proportion } \\
\text { d'invendus }\end{array}$ \\
\hline Allier ..... & 1192 & $15 \%$ & 25767 & $6 \%$ \\
\hline Loir-et-Cher.... & 715 & $-15 \%$ & 12345 & $3 \%$ \\
\hline Indre ............. & 1171 & $6 \%$ & 24824 & $2 \%$ \\
\hline Sarthe ....... & 1176 & $11 \%$ & 13367 & $0 \%$ \\
\hline Orne ..... & 863 & $16 \%$ & 16689 & $5 \%$ \\
\hline Nièvre ........... & 782 & $5 \%$ & 24617 & $4 \%$ \\
\hline 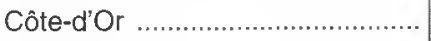 & 791 & $13 \%$ & 33548 & $4 \%$ \\
\hline Haute-Marne ..... & 671 & $11 \%$ & 28250 & $11 \%$ \\
\hline Haute-Saône ............................... & 750 & $14 \%$ & 63899 & $4 \%$ \\
\hline
\end{tabular}

Tableau IV

Chêne de 30 à $45 \mathrm{~cm}$ de diamètre

Principaux départements producteurs - Grandes ventes 1994

\begin{tabular}{|c|c|c|c|c|}
\hline Départements & $\begin{array}{l}\text { Prix moyen } \\
1994\left(\mathrm{~F} / \mathrm{m}^{3}\right)\end{array}$ & $\begin{array}{c}\text { Variation } \\
1994 / 1993\end{array}$ & $\begin{array}{l}\text { Volume vendu } \\
\qquad\left(\mathrm{m}^{3}\right)\end{array}$ & $\begin{array}{l}\text { Proportion } \\
\text { d'invendus }\end{array}$ \\
\hline Allier & 293 & $11 \%$ & 19567 & $6 \%$ \\
\hline Loir-et-Cher. & 220 & $10 \%$ & 19909 & $1 \%$ \\
\hline Indre ............ & 228 & $-11 \%$ & 10480 & $11 \%$ \\
\hline Sarthe ........ & 249 & $20 \%$ & 12617 & $0 \%$ \\
\hline Orne ........ & 279 & $7 \%$ & 24461 & $3 \%$ \\
\hline ................. & 207 & $-13 \%$ & 16734 & $7 \%$ \\
\hline Côte-d'Or ..................................... & 221 & $7 \%$ & 19062 & $5 \%$ \\
\hline 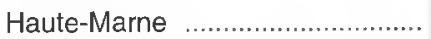 & 180 & $13 \%$ & 20371 & $19 \%$ \\
\hline Haute-Saône ................................. & 238 & $8 \%$ & 30345 & $5 \%$ \\
\hline
\end{tabular}

\section{LE HÊTRE}

La demande en a été particulièrement vive, les invendus quasi inexistants et les prix en hausse marquée sur l'automne 1993 (+ $29 \%$ en moyenne), se fixant ainsi à un niveau supérieur à celui de 1989 (cf. diagramme 2 et graphique 4, p. 314).

En effet, cette essence est l'objet depuis quelques années d'un intérêt marqué : il se substitue à certains emplois de bois tropicaux; il peut désormais se trancher comme le Chêne ; il bénéficie d'un effet de mode de l'ameublement pour les bois clairs.

Tous ces effets conjugués ont entraîné une baisse moindre que pour les autres essences pendant les trois années de crise et une hausse vigoureuse en 1994.

On notera plus particulièrement le haut niveau du prix moyen de cette essence en Haute-Saône, allié à la quasi-inexistence des invendus (cf. tableau V, p. 314). 


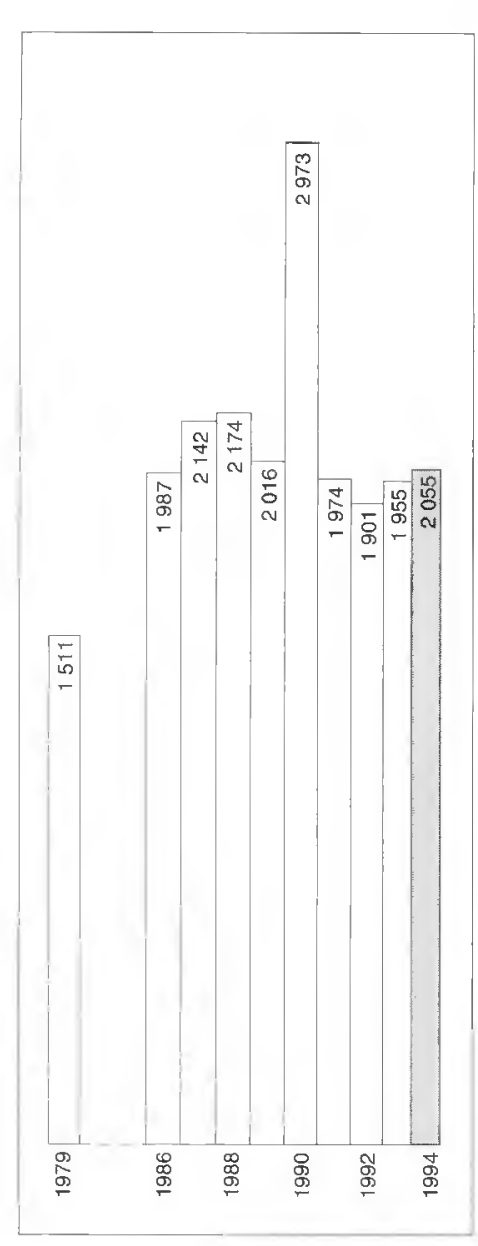

Diagramme 2

HÊTRE. VOLUMES-GRUME MOBILISÉS EN FORÊT SOUMISE (en $\left.1000 \mathrm{~m}^{3}\right)$
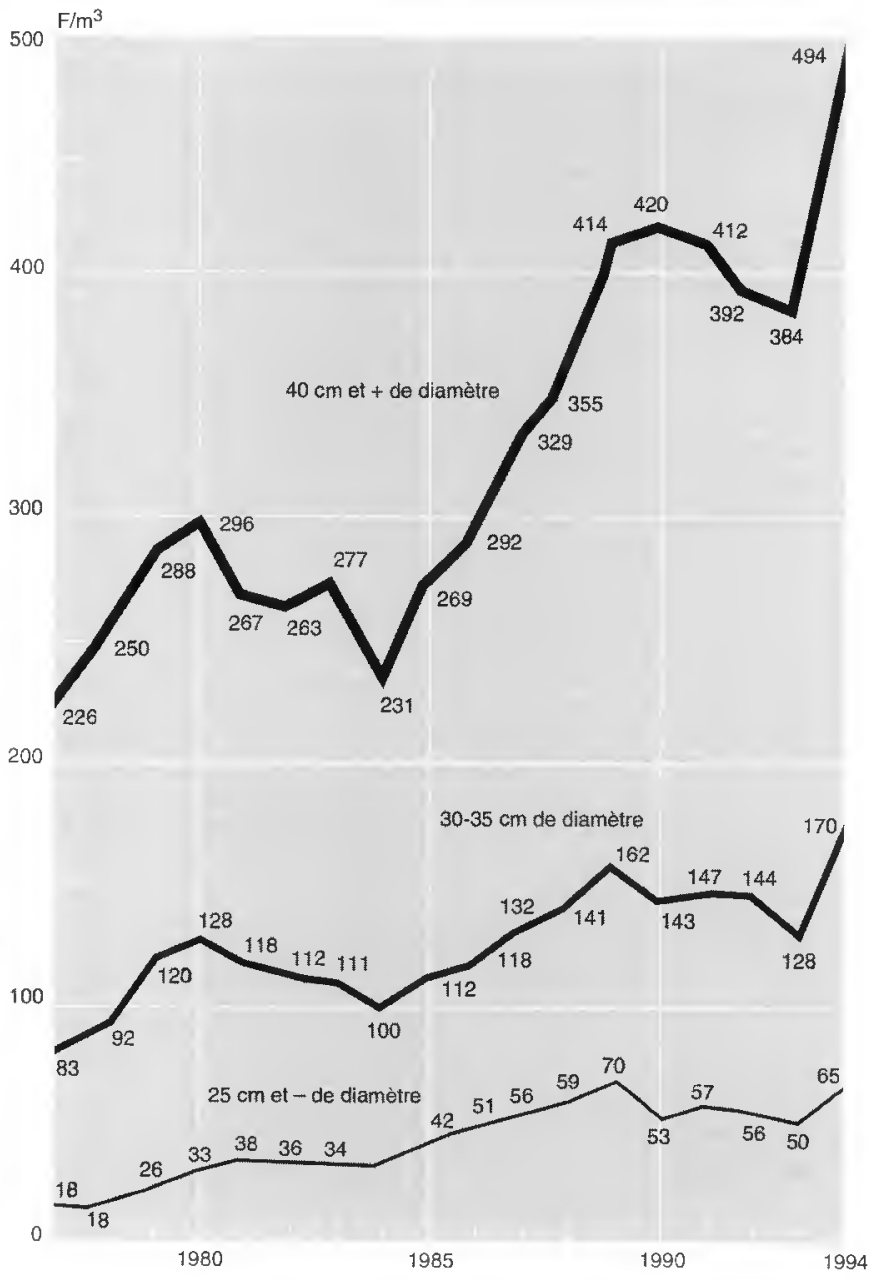

Graphique 4 HÊTRE. ÉVOLUTION DES PRIX MOYENS AUX GRANDES VENTES

Tableau $\mathrm{V}$

Hêire de $40 \mathrm{~cm}$ et plus de diamètre

Principaux départements producteurs - Grandes ventes 1994

\begin{tabular}{|c|c|c|c|c|}
\hline Départements & $\begin{array}{l}\text { Prix moyen } \\
1994\left(\mathrm{~F} / \mathrm{m}^{3}\right)\end{array}$ & $\begin{array}{c}\text { Variation } \\
1994 / 1993\end{array}$ & $\begin{array}{l}\text { Volume vendu } \\
\qquad\left(\mathrm{m}^{3}\right)\end{array}$ & $\begin{array}{l}\text { Proportion } \\
\text { d'invendus }\end{array}$ \\
\hline Eure/Seine-Maritime ..................... & 509 & $21 \%$ & 126886 & $4 \%$ \\
\hline 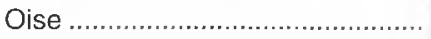 & 445 & $23 \%$ & 21956 & $2 \%$ \\
\hline 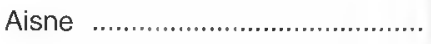 & 482 & $23 \%$ & 17848 & $2 \%$ \\
\hline Haute-Marne........................... & 601 & $19 \%$ & 37180 & $8 \%$ \\
\hline Meuse ......................................... & 543 & $24 \%$ & 54422 & $4 \%$ \\
\hline Pyrénées-Atlantiques .................. & 363 & $27 \%$ & 18780 & $21 \%$ \\
\hline Haute-Saône ................................ & 694 & $21 \%$ & 51761 & $1 \%$ \\
\hline
\end{tabular}




\section{LES AUTRES FEUILLUS}

Il est toujours très difficile d'analyser cette rubrique qui regroupe des produits extrêmement hétérogènes destinés à des utilisations très diverses comme la trituration ou la caisserie (Bouleau, Charmes, Tremble,...), le placage, l'ébénisterie ou la belle menuiserie (Alisier torminal, Érable, Frêne, Merisier,...).

Très demandés en 1994, tant pour la trituration que pour des utilisations plus nobles (diagramme 3 , ci-dessous), les prix suivent la tendance générale.

Parmi les essences précieuses, le Merisier a paru particulièrement recherché.

\section{Diagramme 3 \\ AUTRES \\ FEUILLUS. \\ VOLUMES- \\ GRUME \\ MOBILISÉS \\ EN FORÊT \\ SOUMISE \\ (en $\left.1000 \mathrm{~m}^{3}\right)$}

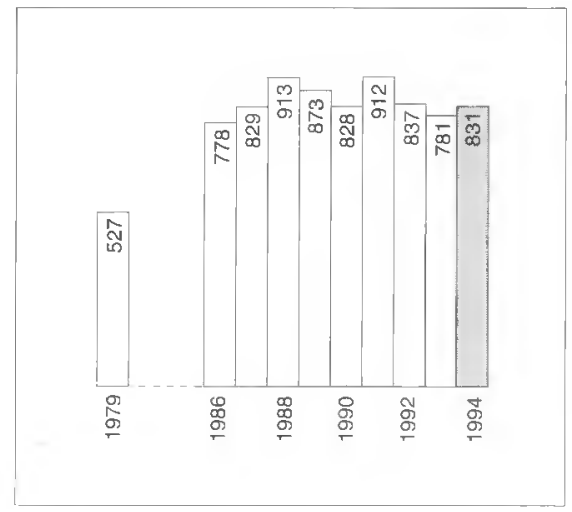

\section{LE SAPIN-ÉPICÉA}

Les quantités mobilisées en ces essences ont été très élevées (diagramme 4, ci-contre), se rapprochant du niveau historique de 1990 (chablis).

Dès le printemps, la reprise de la demande avait entraîné une revalorisation des prix de 10 à $15 \%$ sur l'automne précédent. Cette hausse s'est poursuivie à l'automne pour atteindre finalement plus de $25 \%$ en moyenne sur un an. Toutefois, cette forte hausse ne permet même pas de retrouver le niveau des prix de 1991 en francs courants (cf. graphique 5, p. 316).

Diagramme 4

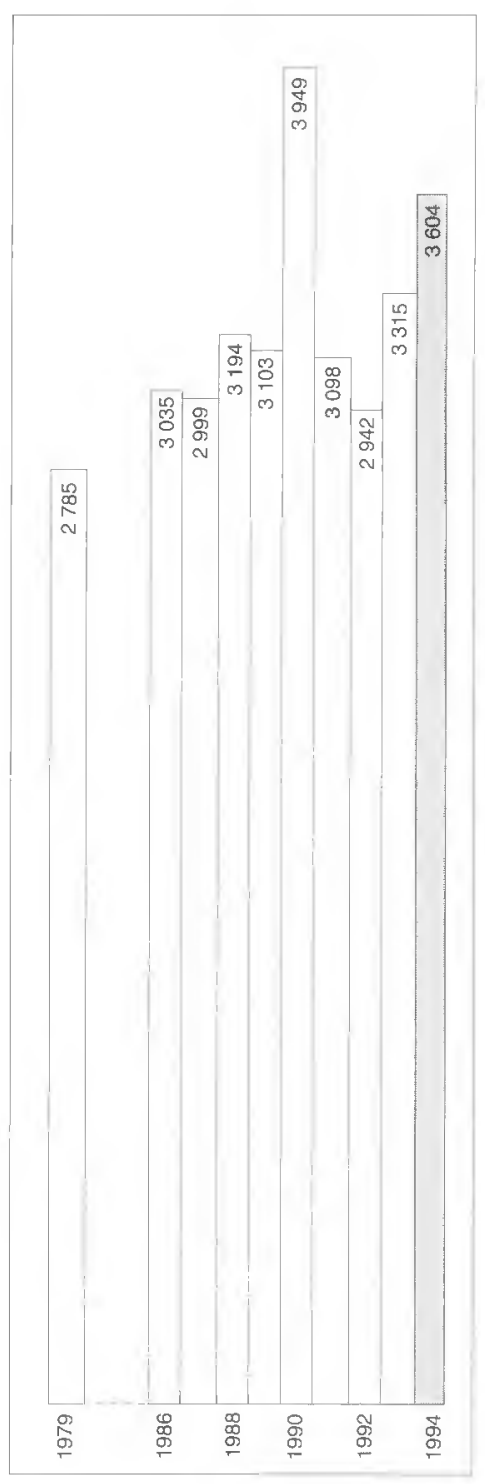




\section{OFFICE NATIONAL DES FORÊTS}

Graphique 5

$400 \mathrm{~F} / \mathrm{m}^{3}$

SAPIN-ÉPICÉA.

ÉVOLUTION DES PRIX MOYENS AUX GRANDES VENTES

La hausse est apparemment moindre dans la moitié Sud de la France comparativement au quart Nord-Est; mais il convient de tempérer cette observation dans la mesure où la commercialisation, dans les régions de haute montagne, de coupes d'exploitation et de vidange difficile, traditionnellement invendues dès lors que le marché est peu porteur, pèse sur les prix moyens des départements concernés. La diminution très sensible des invendus (même si ceux-ci restent encore conséquents dans ces départements de haute montagne) en témoigne clairement (ct. tableau VI).

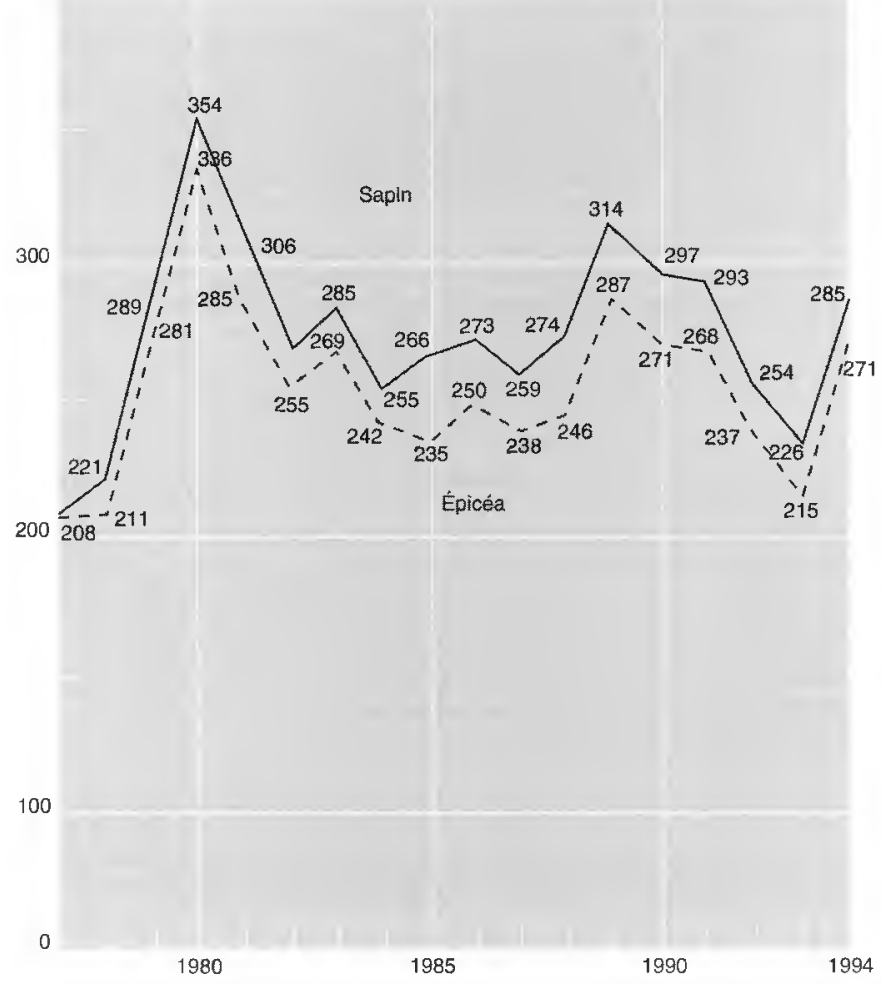

Tableau VI

Sapin et Épicéa de plus de $25 \mathrm{~cm}$ de diamètre

Principaux départements producteurs - Grandes ventes 1994

\begin{tabular}{|c|c|c|c|c|c|}
\hline Départements & Essences & $\begin{array}{l}\text { Prix moyen } \\
1994\left(\mathrm{~F} / \mathrm{m}^{3}\right)\end{array}$ & $\begin{array}{c}\text { Variation } \\
1994 / 1993\end{array}$ & $\begin{array}{l}\text { Volume vendu } \\
\qquad\left(\mathrm{m}^{3}\right)\end{array}$ & $\begin{array}{l}\text { Proportion } \\
\text { d'invendus }\end{array}$ \\
\hline 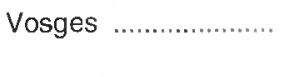 & $\begin{array}{l}\text { Sapin } \\
\text { Épicéa }\end{array}$ & $\begin{array}{l}343 \\
377\end{array}$ & $\begin{array}{l}29 \% \\
34 \%\end{array}$ & $\begin{array}{r}170936 \\
51595\end{array}$ & $\begin{array}{l}5 \% \\
4 \%\end{array}$ \\
\hline Doubs …........................ & $\begin{array}{l}\text { Sapin } \\
\text { Épicéa }\end{array}$ & $\begin{array}{l}325 \\
375\end{array}$ & $\begin{array}{l}19 \% \\
20 \%\end{array}$ & $\begin{array}{r}114216 \\
77946\end{array}$ & $\begin{array}{l}1 \% \\
1 \%\end{array}$ \\
\hline Jura & $\begin{array}{l}\text { Sapin } \\
\text { Épicéa }\end{array}$ & $\begin{array}{l}293 \\
346\end{array}$ & $\begin{array}{l}26 \% \\
25 \%\end{array}$ & $\begin{array}{l}80694 \\
28426\end{array}$ & $\begin{array}{l}0 \% \\
0 \%\end{array}$ \\
\hline Ain & $\begin{array}{l}\text { Sapin } \\
\text { Épicéa }\end{array}$ & $\begin{array}{l}266 \\
321\end{array}$ & $\begin{array}{l}19 \% \\
21 \%\end{array}$ & $\begin{array}{l}70087 \\
22634\end{array}$ & $\begin{array}{l}3 \% \\
1 \%\end{array}$ \\
\hline Haute-Savoie ............ & $\begin{array}{l}\text { Sapin } \\
\text { Épicéa }\end{array}$ & $\begin{array}{l}221 \\
227\end{array}$ & $\begin{array}{l}6 \% \\
0 \%\end{array}$ & $\begin{array}{r}8729 \\
20056\end{array}$ & $\begin{array}{l}32 \% \\
33 \%\end{array}$ \\
\hline Savoie & $\begin{array}{l}\text { Sapin } \\
\text { Épicéa }\end{array}$ & $\begin{array}{l}247 \\
230\end{array}$ & $\begin{array}{l}20 \% \\
13 \%\end{array}$ & $\begin{array}{l}25500 \\
43961\end{array}$ & $\begin{array}{l}24 \% \\
23 \%\end{array}$ \\
\hline Aude .................. & Sapin & 318 & $14 \%$ & 14262 & $14 \%$ \\
\hline Hautes-Pyrénées ..... & Sapin & 247 & $12 \%$ & 18578 & $11 \%$ \\
\hline Puy-de-Dôme ............. & $\begin{array}{l}\text { Sapin } \\
\text { Épicéa }\end{array}$ & $\begin{array}{l}274 \\
224\end{array}$ & $\begin{array}{l}0 \% \\
0 \%\end{array}$ & $\begin{array}{l}19241 \\
12384\end{array}$ & $\begin{array}{l}7 \% \\
7 \%\end{array}$ \\
\hline
\end{tabular}




\section{LE PIN SYLVESTRE}

L'examen du diagramme 5 reflète bien la diminution des disponibilités en Pin sylvestre annoncée depuis de nombreuses années.

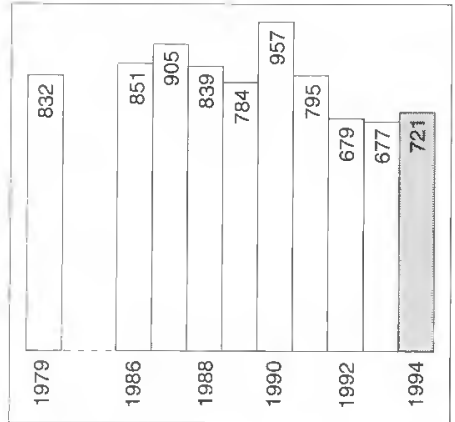

Diagramme 5

PIN SYLVESTRE, VOLUMES-GRUME MOBILISÉS EN FORÊT SOUMISE

(en $\left.1000 \mathrm{~m}^{3}\right)$

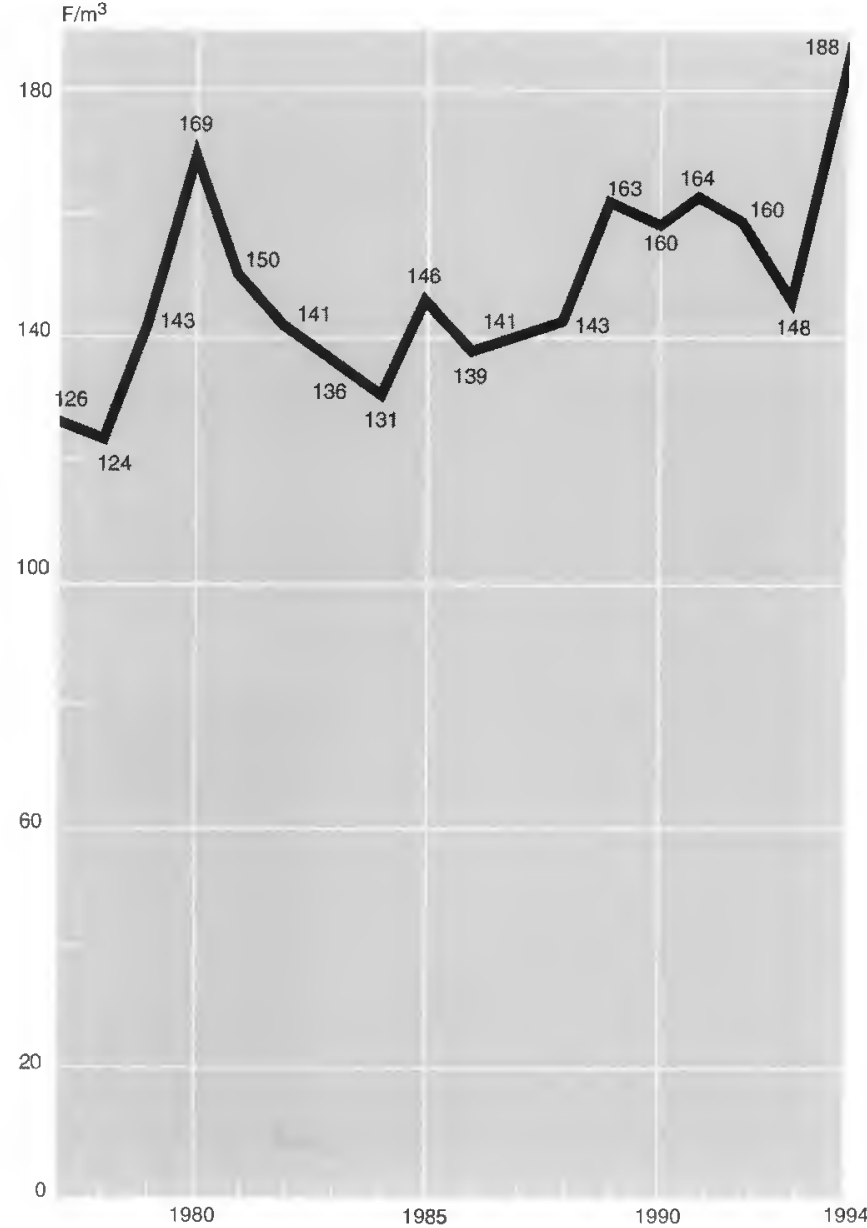

Tableau VII

Pin sylvestre de plus de $25 \mathrm{~cm}$ de diamètre

Principaux départements producteurs - Grandes ventes 1994

\begin{tabular}{|c|c|c|c|c|}
\hline Départements & $\begin{array}{l}\text { Prix moyen } \\
1994\left(\mathrm{~F} / \mathrm{m}^{3}\right)\end{array}$ & $\begin{array}{c}\text { Variation } \\
1994 / 1993\end{array}$ & $\begin{array}{l}\text { Volume vendu } \\
\left(\mathrm{m}^{3}\right)\end{array}$ & $\begin{array}{l}\text { Proportion } \\
\text { d'invendus }\end{array}$ \\
\hline Loiret ............... & 263 & $29 \%$ & 46000 & $0 \%$ \\
\hline Seine-et-Marne & 152 & $34 \%$ & 14642 & $2 \%$ \\
\hline 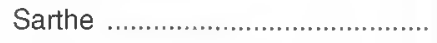 & 238 & $26 \%$ & 7544 & $0 \%$ \\
\hline Orne $\ldots . . .$. & 306 & $14 \%$ & 6213 & $11 \%$ \\
\hline Eure/Seine-Maritime ..................... & 228 & $14 \%$ & 26536 & $5 \%$ \\
\hline Puy-de-Dôme ...... & 234 & $6 \%$ & 14465 & $6 \%$ \\
\hline Haute-Loire .... & 184 & $11 \%$ & 8553 & $7 \%$ \\
\hline Vosges ............. & 202 & $23 \%$ & 27270 & $16 \%$ \\
\hline Bretagne ...... & 205 & $-1 \%$ & 7215 & $3 \%$ \\
\hline
\end{tabular}




\section{OFFICE NATIONAL DES FORẼTS}

La demande étant très vive en 1994, mais l'offre étant plafonnée, les volumes vendus r'ont pu augmenter sur l'an passé que dans la mesure de la diminution des invendus (assez lourds en 1993).

La hausse moyenne $(+26 \%$ ) des prix a été du même ordre que pour le Sapin-Épicéa (cf. graphique 6, p. 317) ; le prix moyen est toutetois le plus haut, en francs courants, de ceux des dernières années.

A noter tout particulièrement l'absence d'invendus et le niveau élevé du prix moyen dans le département le plus producteur, le Loiret (cf. tableau VII, p. 317).

\section{LE PIN MARITIME}

La récolte de Pin maritime en forêt publique a toujours été en dents de scie comme en témoigne le diagramme 6, ci-dessous; mais la forêt publique n'a qu'une importance très relative, comparée à la forêt privée, en Aquitaine.

Des invendus beaucoup moins importants que d'habitude et une hausse moyenne de plus de $35 \%$ (ct. graphique 7 , ci-dessous) font état de la réactivation du marché.

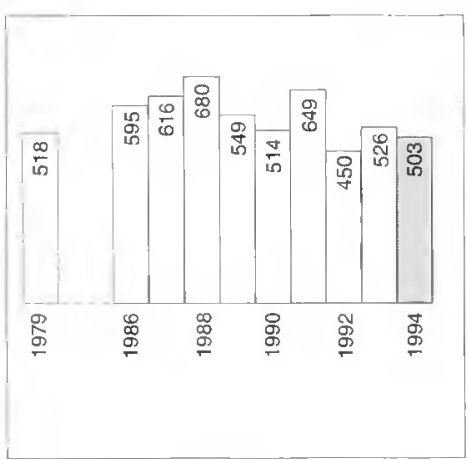

Diagramme 6

PIN MARITIME. VOLUMES-GRUME MOBILISÉS EN FORÊT SOUMISE (en $1000 \mathrm{~m}^{3}$ )

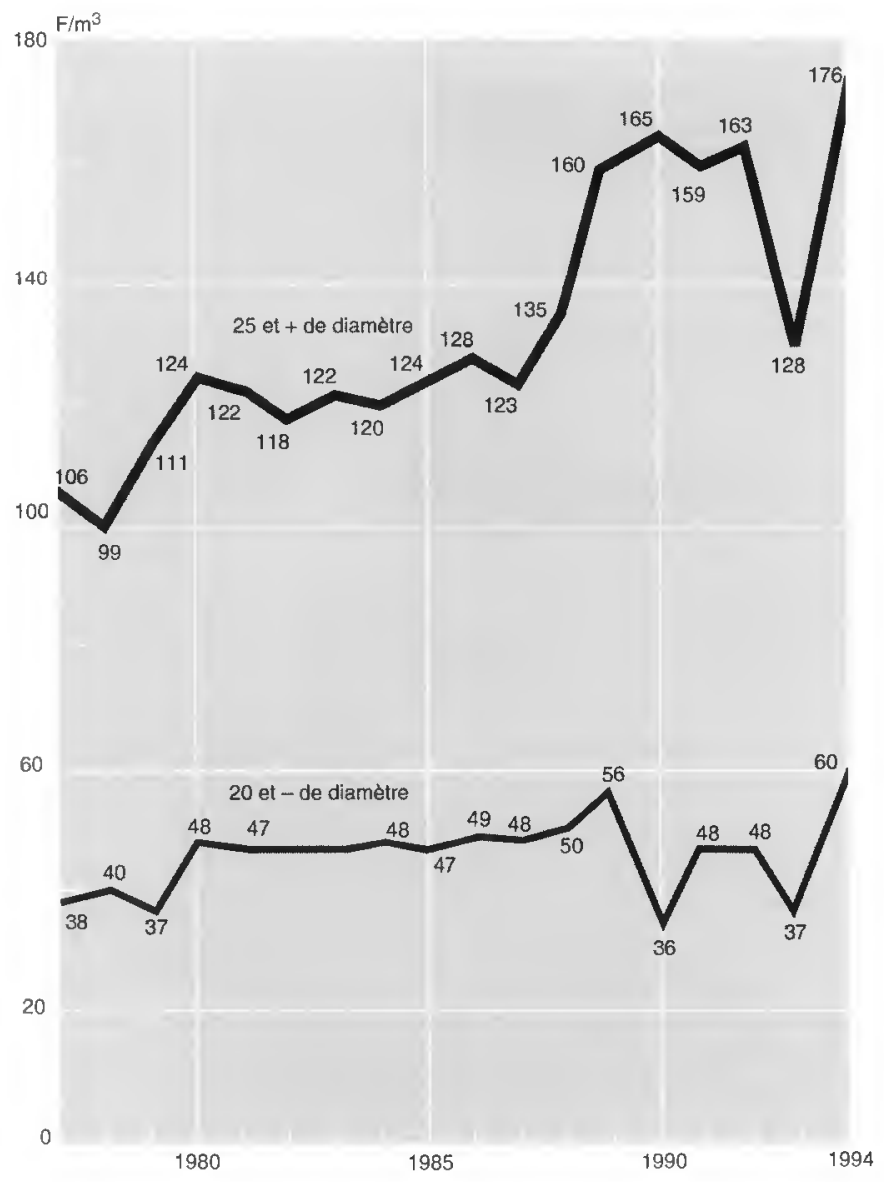


Pin maritime de plus de $25 \mathrm{~cm}$ de diamètre

Principaux départements producteurs - Grandes ventes 1994

\begin{tabular}{|c|c|c|c|c|}
\hline Départements & $\begin{array}{c}\text { Prix moyen } \\
1994\left(\mathrm{~F} / \mathrm{m}^{3}\right)\end{array}$ & $\begin{array}{c}\text { Variation } \\
1994 / 1993\end{array}$ & $\begin{array}{c}\text { Volume vendu } \\
\left(\mathrm{m}^{3}\right)\end{array}$ & $\begin{array}{c}\text { Proportion } \\
\text { d'invendus }\end{array}$ \\
\hline Aquitaine ..................................... & 181 & $30 \%$ & 92574 & $38 \%$ \\
Poitou-Charentes .......................... & 144 & $-1 \%$ & 12658 & $5 \%$ \\
Pays-de-Loire ............................... & 147 & $18 \%$ & 11425 & $0 \%$ \\
\hline
\end{tabular}

\section{LES AUTRES RÉSINEUX}

L'offre ne cesse de s'accroître d'année en année. Les invendus, toujours importants dans les régions du Sud-Est, se sont un peu réduits et les prix ont accusé une hausse apparemment plus modérée que celle enregistrée sur les essences résineuses visées plus haut. La relative diminution des invendus $a$, en effet, pesé sur le prix moyen.

Diagramme 7

AUTRES RÉSINEUX.

VOLUMES-GRUME MOBILISÉS EN FORÊT SOUMISE

(en $1000 \mathrm{~m}^{3)}$

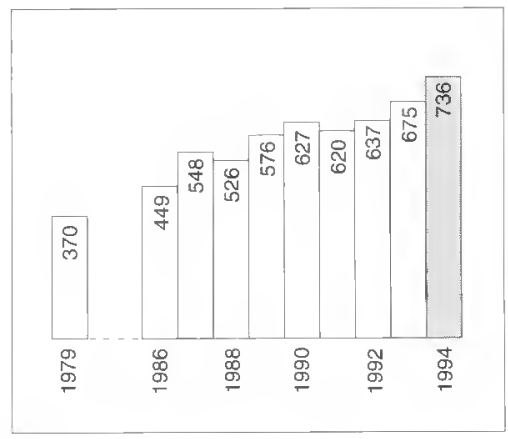

Tableau IX

Résineux divers tous diamètres

\begin{tabular}{|c|c|c|c|c|}
\hline & $\begin{array}{l}\text { Prix moyen } \\
1994\left(\mathrm{~F} / \mathrm{m}^{3}\right)\end{array}$ & $\begin{array}{l}\text { Variation } \\
1994 / 1993\end{array}$ & $\begin{array}{l}\text { Volume vendu } \\
\qquad\left(\mathrm{m}^{3}\right)\end{array}$ & $\begin{array}{l}\text { Proportion } \\
\text { d'invendus }\end{array}$ \\
\hline $\begin{array}{l}\text { Pin noir : } \\
\quad \text { PACA (1) } \\
\text { Lozère }\end{array}$ & $\begin{array}{r}74 \\
153\end{array}$ & $\begin{array}{l}+23 \% \\
+15 \%\end{array}$ & $\begin{array}{l}24000 \\
15700\end{array}$ & $\begin{array}{r}18 \% \\
4 \%\end{array}$ \\
\hline $\begin{array}{l}\text { Mélèze : } \\
\text { PACA }\end{array}$ & 175 & $+5 \%$ & 9000 & $65 \%$ \\
\hline $\begin{array}{l}\text { Pin laricio: } \\
\quad \text { Corse }\end{array}$ & 307 & $+13 \%$ & 18900 & $40 \%$ \\
\hline $\begin{array}{l}\text { Pin à crochets : } \\
\text { PACA }\end{array}$ & 43 & $+13 \%$ & 1700 & $73 \%$ \\
\hline $\begin{array}{l}\text { Pin d'Alep: } \\
\text { PACA }\end{array}$ & 48 & $+2 \%$ & 2900 & $17 \%$ \\
\hline $\begin{array}{l}\text { Cèdre : } \\
\text { PACA . }\end{array}$ & 138 & n.s. & 900 & $55 \%$ \\
\hline $\begin{array}{l}\text { Douglas : } \\
\quad \text { Rhône }\end{array}$ & 223 & $+26 \%$ & 4500 & $22 \%$ \\
\hline
\end{tabular}

(1) Provence-Alpes-Côte d'Azur. 


\section{OFFICE NATIONAL DES FORÊTS}

\section{LES PETITS BOIS}

Feuillus comme résineux, les petits bois ont été très demandés, notamment pour l'approvisionnement des industries de la pâte à papier.

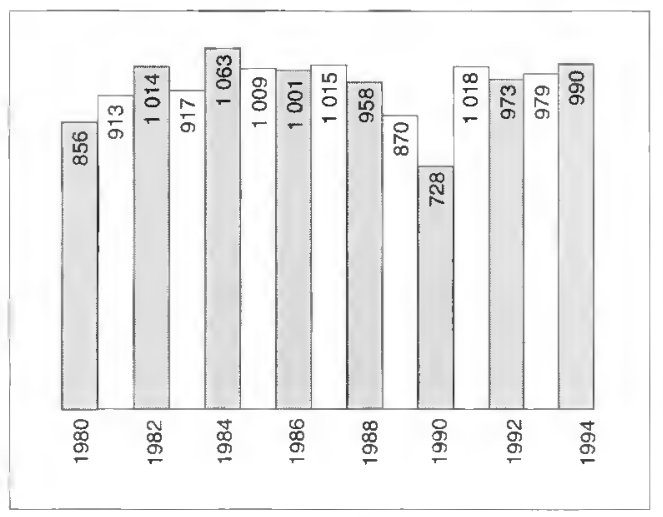

Diagramme 8

TAILLIS.

VOLUMES VENDUS ET DÉLIVRÉS DE 1980 À 1994 (en $1000 \mathrm{~m}^{3}$ )

\section{CONCLUSIONS}

L'année 1994 a donc été marquée par un niveau record de la récolte (hors chablis exceptionnels de 1990 ) et par une certaine revalorisation des prix des bois après quatre années de baisse consécutive. Néanmoins, en francs constants et en longue période, ces prix se situent dans la moyenne en matière de feuillus et toujours au-dessous de la moyenne en matière de résineux (cf. annexes $3 \mathrm{~A}$ et $3 B, p .322)$.

L'importance des volumes offerts et mobilisés en forêt publique témoigne bien du rôle capital de l'Office national des Forêts dans la filière française du bois. Ce rôle concerne tant la régularité des approvisionnements que l'adaptation des modes de mise en marché aux besoins des clients : étalement des ventes dans le temps et développement des contrats pluriannuels d'approvisionnement, des contrats négociés, etc. 
ANNEXE 1 RÉSULTATS NATIONAUX DES VENTES D'AUTOMNE 1994 (en francs courants)

\begin{tabular}{|c|c|c|c|c|c|}
\hline $\begin{array}{c}\text { Essences } \\
\text { Volumes-tiges } \\
\text { diamètre à } 1,30 \mathrm{~m} \text { du sol }\end{array}$ & $\begin{array}{c}\text { Prix moyen } \\
1989 \\
\left(\mathrm{~F} / \mathrm{m}^{3}\right)\end{array}$ & $\begin{array}{c}\text { Prix moyen } \\
1991 \\
\left(\mathrm{~F} / \mathrm{m}^{3}\right)\end{array}$ & $\begin{array}{c}\text { Prix moyen } \\
1993 \\
\left(\mathrm{~F} / \mathrm{m}^{3}\right)\end{array}$ & $\begin{array}{c}\text { Prix moyen } \\
1994 \\
\left(\mathrm{~F} / \mathrm{m}^{3}\right) \\
\end{array}$ & $\begin{array}{l}\text { Variation } \\
1994-1993\end{array}$ \\
\hline Chêne $50 \mathrm{~cm}$ et..+ & 867,0 & 765,0 & 680,0 & 758,0 & $+11 \%$ \\
\hline Chêne $30-45 \mathrm{~cm}$ & 261,0 & 233,0 & 198,0 & 226,0 & $+14 \%$ \\
\hline Chêne $25 \mathrm{~cm}$ et $-\ldots \ldots \ldots \ldots \ldots$ & 54,0 & 50,0 & 54,0 & 69,0 & $+28 \%$ \\
\hline TOTAL CHÊNE & 542,0 & 485,0 & 432,0 & 484,0 & $+12 \%$ \\
\hline Hêtre $40 \mathrm{~cm}$ et $+\ldots \ldots \ldots \ldots \ldots$ & 414,0 & 412,0 & 384,0 & 494,0 & $+29 \%$ \\
\hline Hêtre $30-35 \mathrm{~cm}$ & 162,0 & 147,0 & 128,0 & 170,0 & $+33 \%$ \\
\hline Hêtre $25 \mathrm{~cm}$ et $-\ldots \ldots \ldots \ldots \ldots$ & 70,0 & 57,0 & 50,0 & 64,0 & $+28 \%$ \\
\hline TOTAL HÊTRE & 328,0 & 324,0 & 305,0 & 392,0 & $+29 \%$ \\
\hline $\begin{array}{l}\text { Sapin } 25 \mathrm{~cm} \text { et }+\ldots \ldots \ldots \ldots \ldots \ldots \\
\text { Sapin } 20 \mathrm{~cm} \text { et }-\ldots \ldots \ldots \ldots \ldots \ldots \ldots\end{array}$ & $\begin{array}{r}326,0 \\
43,0\end{array}$ & $\begin{array}{r}304,0 \\
41,0\end{array}$ & $\begin{array}{r}233,0 \\
29,0\end{array}$ & $\begin{array}{r}293,0 \\
37,0\end{array}$ & $\begin{array}{l}+26 \% \\
+28 \%\end{array}$ \\
\hline TOTAL SAPIN .......................... & 314,0 & 293,0 & 226,0 & 285,0 & $+26 \%$ \\
\hline Épicéa $25 \mathrm{~cm}$ et $+\ldots \ldots \ldots \ldots \ldots$ & 317,0 & 291,0 & 233,0 & 292,0 & $+25 \%$ \\
\hline Épicéa $20 \mathrm{~cm}$ et $-\ldots \ldots \ldots \ldots \ldots . .$. & 58,0 & 51,0 & 37,0 & 48,0 & $+30 \%$ \\
\hline TOTAL ÉPICÉA & 287,0 & 268,0 & 215,0 & 271,0 & $+26 \%$ \\
\hline Pin sylvestre $25 \mathrm{~cm}$ et $+\ldots \ldots$ & 182,0 & 182,0 & 163,0 & 205,0 & $+26 \%$ \\
\hline Pin sylvestre $20 \mathrm{~cm}$ et $-\ldots \ldots$ & 27,0 & 27,0 & 28,0 & 36,0 & $+29 \%$ \\
\hline TOTAL PIN SYLVESTRE .... & 163,0 & 164,0 & 148,0 & 188,0 & $+27 \%$ \\
\hline $\begin{array}{l}\text { Pin maritime } 25 \mathrm{~cm} \text { et }+\ldots \ldots . . \\
\text { Pin maritime } 20 \mathrm{~cm} \text { et }-\ldots \ldots .\end{array}$ & $\begin{array}{r}160,0 \\
56,0\end{array}$ & $\begin{array}{r}159,0 \\
48,0\end{array}$ & $\begin{array}{r}128,0 \\
37,0\end{array}$ & $\begin{array}{r}176,0 \\
60,0\end{array}$ & $\begin{array}{l}+38 \% \\
+62 \%\end{array}$ \\
\hline TOTAL PIN MARITIME ........ & 143,0 & 140,0 & 115,0 & 156,0 & $+36 \%$ \\
\hline Taillis feuillus ............................ & 45,0 & 46,0 & 42,0 & 55,0 & $+31 \%$ \\
\hline $\begin{array}{l}\text { Toutes essences } \\
\text { et toutes catégories ............... }\end{array}$ & 259,0 & 246,0 & 221,0 & 271,0 & $+23 \%$ \\
\hline
\end{tabular}

ANNEXE 2

L'ÉVOLUTION DE L'OFFRE DE BOIS DE L'ONF

\begin{tabular}{|c|c|c|c|c|}
\hline$\left(\right.$ En $\left.1000 \mathrm{~m}^{3}\right)$ & Volume mobilisé & $\begin{array}{c}\text { Offre de bois } \\
\text { frais }\end{array}$ & $\begin{array}{c}\text { Invendus } \\
\text { au } 31 / 12\end{array}$ & $\begin{array}{c}\text { Offre } \\
\text { totale }\end{array}$ \\
\hline 1980 & 10476 & 10876 & & 11332 \\
1981 & 10473 & 11067 & 1450 & 11923 \\
1982 & 11298 & 11295 & 1447 & 12745 \\
1983 & 12687 & 12405 & 1165 & 13852 \\
1984 & 13406 & 13613 & 1372 & 14778 \\
1985 & 13410 & 13390 & 1352 & 14762 \\
1986 & 13068 & 12941 & 1225 & 14293 \\
1987 & 13427 & 13284 & 1082 & 14509 \\
1988 & 14015 & 13988 & 1055 & 15070 \\
1989 & 13467 & 13295 & 883 & 14350 \\
1990 & 15800 & 16347 & 1430 & 17230 \\
1991 & 13785 & 14015 & 1660 & 15445 \\
1992 & 13050 & 13239 & 1849 & 14899 \\
1993 & 13386 & 13145 & 1608 & 14994 \\
1994 & 14065 & 13247 & 790 & 14855 \\
\hline
\end{tabular}


ANNEXE 3A ÉVOLUTION DU PRIX MOYEN DU CHÊNE ET DU HÊTRE DE 1966 À 1994 (en francs constants)
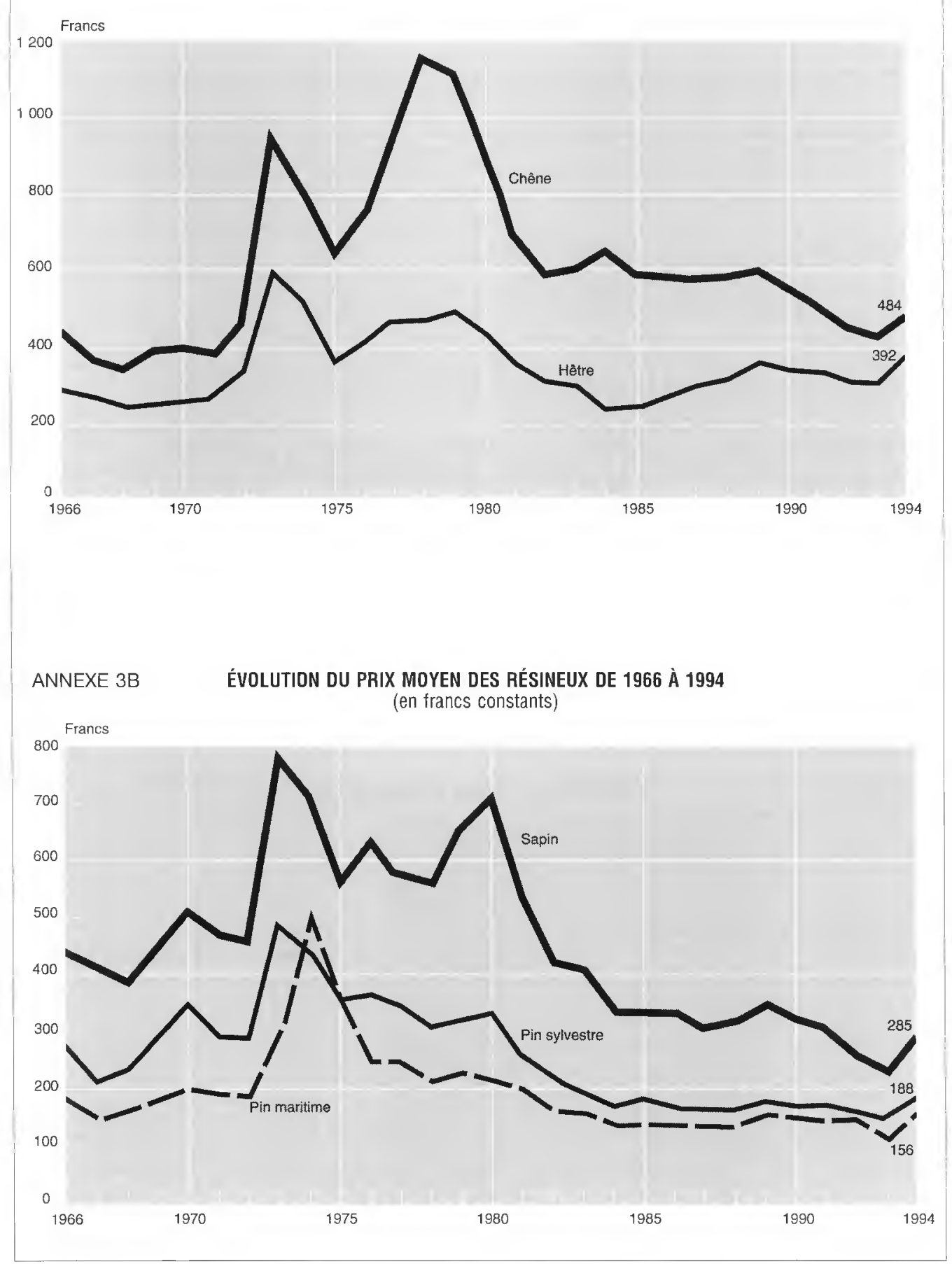


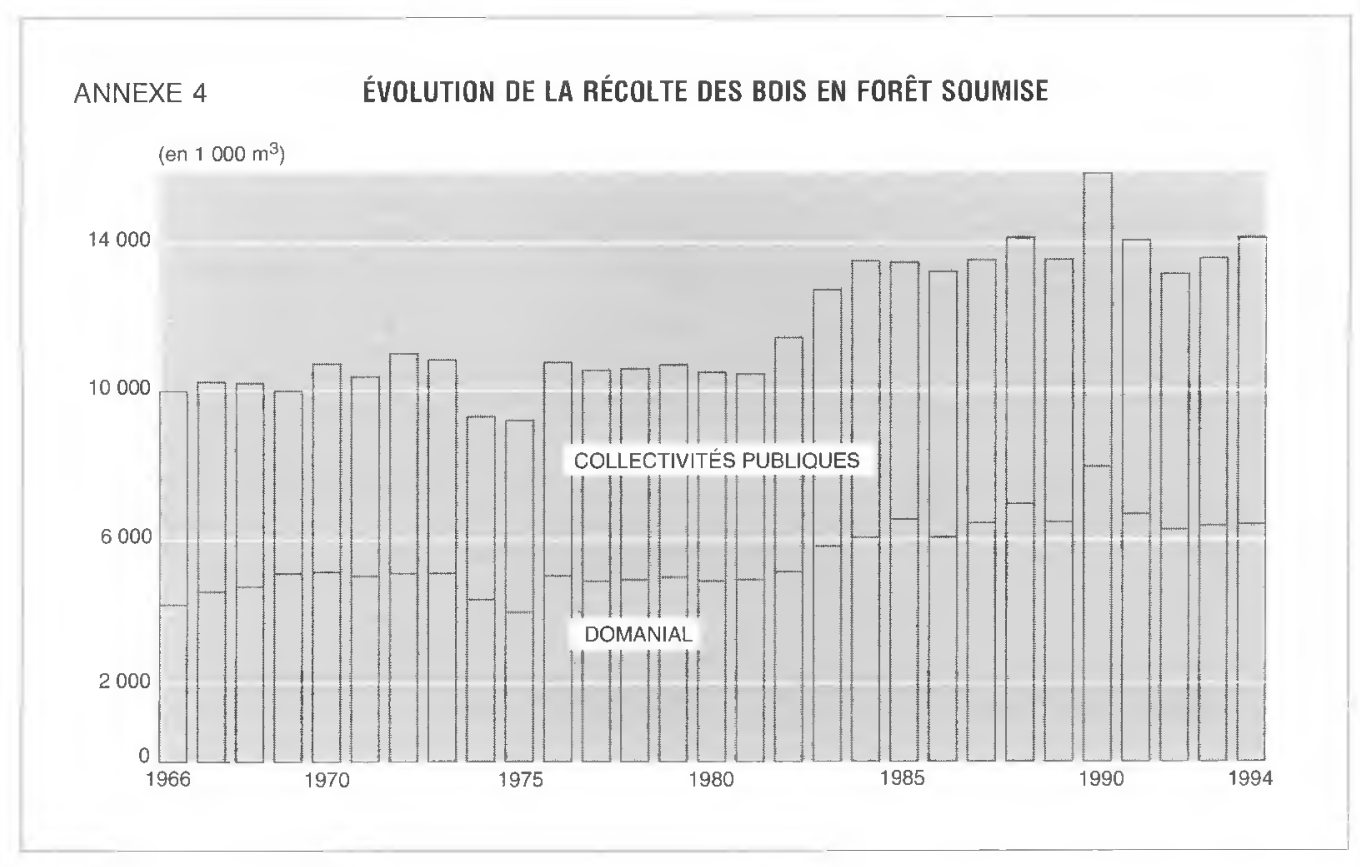

ANNEXE 5

ONF - ÉVOLUTION DES MODES DE MISE EN MARCHÉ

\begin{tabular}{|c|c|c|c|c|c|c|}
\hline & 1970 & 1980 & 1989 & 1992 & 1993 & 1994 \\
\hline Récolte & $10,8 \mathrm{Mm}^{3}$ & $10,5 \mathrm{Mm}^{3}$ & $13,5 \mathrm{Mm}^{3}$ & $13,0 \mathrm{Mm}^{3}$ & $13,4 \mathrm{Mm}^{3}$ & $14,1 \mathrm{Mm}^{3}$ \\
\hline Coupes sur pied & $84 \%$ & $84 \%$ & $84 \%$ & $83 \%$ & $82 \%$ & $82 \%$ \\
\hline Bois façonnés & $16 \%$ & $16 \%$ & $16 \%$ & $17 \%$ & $18 \%$ & $18 \%$ \\
\hline Délivrances & $13 \%$ & $16 \%$ & $12 \%$ & $11 \%$ & $10 \%$ & $11 \%$ \\
\hline Ventes amiables & $8 \%$ & $9 \%$ & $12 \%$ & $16 \%$ & $17 \%$ & $16 \%$ \\
\hline Adjudications d'automne ... & $58 \%$ & $48 \%$ & $45 \%$ & $40 \%$ & $38 \%$ & $39 \%$ \\
\hline Autres ventes $\ldots \ldots \ldots \ldots \ldots \ldots$ & $21 \%$ & $27 \%$ & $31 \%$ & $33 \%$ & $35 \%$ & $34 \%$ \\
\hline $\begin{array}{l}\text { Contrats pluriannuels } \\
\text { d'approvisionnement }\end{array}$ & $75000 \mathrm{~m}^{3}$ & $25000 \mathrm{~m}^{3}$ & $90000 \mathrm{~m}^{3}$ & $230000 \mathrm{~m}^{3}$ & $295000 \mathrm{~m}^{3}$ & $292000 \mathrm{~m}^{3}$ \\
\hline
\end{tabular}


LA MOBILISATION DES BOIS EN PROVENANCE DES FORÊTS BÉNÉFICIANT DU RÉGIME FORESTIER EN 1994 ET LES PRIX OBTENUS AUX GRANDES VENTES DE L'AUTOMNE 1994 (Résumé)

La reprise économique générale n'a pas manqué d'avoir une incidence sur le marché du bois en 1994, se traduisant par une augmentation des volumes vendus et des prix enregistrés.

En effet, les quantités de bais mabilisées en 1994 , en provenance des forêts bénéficiant du régime farestier ${ }_{r}$ se sont chiffrées à 14100 o00 m³ (contre $13380000 \mathrm{~m}^{3}$ en 1993), soit en accroissement de $5 \%$ sur l'année précédente.

La plupart des essences ont connu une hausse de leurs prix moyens globaux : $+12 \%$ pour le Chêne, $+29 \%$ pour le Hêtre, $+25 \%$ pour le Sapin - Épicéa, $+26 \%$ pour le Pin sylvestre, $+35 \%$ pour le Pin maritime.

Une réduction des invendus a été constatée pour presque toutes les essences.

TRADE IN TIMBER FROM THE FORESTS UNDER STATE CONTROL IN 1994 AND PRICES OBTAINS AT THE MAIN SALES OF AUTOMN 1994 (Abstract)

General economic recovery naturally had an effect on the timber market in 1994 leading to an increase in volumes sold and prices recorded. Indeed the quantities of timber traded in 1994 coming from the forests under state control came to $14100000 \mathrm{~m}^{3}$ (compared to $13380000 \mathrm{~m}^{3}$ ), i.e. a $5 \%$ increase over the previous year.

The overall prices for most species increased $:+12 \%$ for oak, $+29 \%$ for beech, $+25 \%$ for fir $/$ spruce, $+26 \%$ for the Scots pine, $+35 \%$ for the maritime pine.

Unsold stocks of nearly all species registered a drop.

DIE HOLZAUFBRINGUNG IN DEN GEMEINDE- UND STAATSWÄLDERN UND DIE BEI DEN GROSSEN HERBSTVERKÄUFEN 1994 ERZ:ELTEN PREISE (Zusammenfassung)

Der allgemeine wirtschaftliche Aufschwung hat seine Auswirkung auf den Holzmarkt 1994 nicht verfehlt, was in einer Zunahme der verkauften Volumen und der Erhöhung der verzeichneten Preise zum Ausdruck kommt.

In der Tat beliefen sich die 1994 aufgebrachten Holzmengen aus den beförsterten Wäldern auf $14100000 \mathrm{~m}^{3}$ (gegen $13380000 \mathrm{~m}^{3}$ im Vorjahr), d.h. ein Zuwachs von $5 \%$ im Vergleich zu 1993.

Die meisten Holzarten haben ihre Durchschnittspreise erhöht $:+12 \%$ für Eiche, $+29 \%$ für Buche $+25 \%$ für Tanne-Fichte, $+26 \%$ für Gemeine Kiefer, + $35 \%$ für Strandkiefer.

Eine Verringerung der unverkauften Holzmenge konnte bei fast allen Holzarten festgestellt werden.

LA MOVILIZACIÓN DE LAS MADERAS PROCEDENTES DE LOS MONTES QUE BENEFICIAN DEL RÉGIMEN FORESTAL EN 1994 Y LOS PRECIOS OBTENIDOS EN LAS GRANDES VENTAS DEL OTOÑO DE 1994 (Resumen)

La recuperación económica general no ha dejado de tener una incidencia en el mercado de la madera en 1994, traducida en un aumento de los volúmenes vendidos y de los precios registrados.

En efecto, las cantidades de madera movilizadas en 1994, procedentes de bosques que beneficiaban del régimen forestal, han sido cifradas en $14100000 \mathrm{~m}^{3}$ (contra $13380000 \mathrm{~m}^{3}$ en 1993), es decir, un crecimiento del $5 \%$ sobre el año precedente.

La mayoría de las variedades han conocida una alza de sus precios medios globales : $+12 \%$ para el Roble, $+29 \%$ para la Haya, $+25 \%$ para el Pino-Epicea, $+26 \%$ para el Pino silvestre, $+35 \%$ para el Pino marítimo.

Una reducción de los invendidos ha sido constatada para casi todas las variedades. 


\section{CALENDRIER \\ DES GRANDES VENTES D'AUTOMNE 1995}

\begin{tabular}{|c|c|c|c|c|}
\hline & Dates & Heures & Lieux de vente & Régions \\
\hline 6 & septembre & 8 h 30 & Bruyères & Lorraine \\
\hline 7 & septembre & $9 \mathrm{~h}$ & Albertville & Rhône-Alpes \\
\hline 12 & septembre & $8 \mathrm{~h} 30$ & Pontarlier 1 & Franche-Comté \\
\hline 12 & septembre & $9 h$ & Châtillon-sur-Seine & Bourgogne \\
\hline 12 & septembre & $10 \mathrm{~h}$ & Rodilhan & Languedoc-Roussillon \\
\hline 13 & septembre & 8 h 30 & Troyes. & Champagne-Ardenne \\
\hline 13 & septembre & $9 \mathrm{~h}$ & Raon-I'Étape & Lorraine \\
\hline 13 & septembre & $9 \mathrm{~h}$ & Grenoble & Rhône-Alpes \\
\hline 14 & septembre & $8 \mathrm{~h}$ & Dole & Franche-Comté \\
\hline 14 & septembre & 8 h 30 & Gap & Prov.-Alpes-C. d'Azur \\
\hline 15 & septembre & $8 \mathrm{~h}$ & Dijon-Plaine & Bourgogne \\
\hline 15 & septembre & 8 h 30 & Nantua & Rhône-Alpes \\
\hline 15 & septembre & $10 \mathrm{~h}$ & Briey & Lorraine \\
\hline 18 & septembre & $9 \mathrm{~h}$ & Lure & Franche-Comté \\
\hline 18 & septembre & 9 ค 30 & Villers-Cotterêts & Picardie \\
\hline 19 & septembre & 8 h 30 & Auxerre & Bourgogne \\
\hline 19 & septembre & 8 h 30 & Neufchâteau & Lorraine \\
\hline 19 & septembre & $9 \mathrm{~h}$ & Chambéry & Rhône-Alpes \\
\hline 20 & septembre & 9 h 30 & Belfort & Franche-Comté \\
\hline 20 & septembre & $10 \mathrm{~h}$ & Nantes & Pays-de-Loire \\
\hline 21 & septembre & 8 h 30 & Saint-Dizier & Champagne-Ardenne \\
\hline 21 & septembre & 8 h 30 & Saint-Claude & Franche-Comté \\
\hline 21 & septembre & $8 \mathrm{~h} 30$ & Digne & Prov.-Alpes-C. d'Azur \\
\hline 21 & septembre & $15 \mathrm{~h}$ & Dax & Aquitaine \\
\hline 22 & septembre & $9 h$ & Grenoble & Rhône-Alpes \\
\hline 22 & septembre & $10 \mathrm{~h}$ & Chartres & Centre \\
\hline 25 & septembre & $8 \mathrm{~h} 30$ & Lunéville & Lorraine \\
\hline 25 & septembre & $9 \mathrm{~h}$ & Dijon-Montagne & Bourgogne \\
\hline 26 & septembre & $8 \mathrm{~h}$ & Gray & Franche-Comté \\
\hline 26 & septembre & $9 h$ & Valence & Rhône-Alpes \\
\hline 26 & septembre & 9 h 30 & Laon & Picardie \\
\hline 27 & septembre & $8 \mathrm{~h}$ & Bar-le-Duc & Lorraine \\
\hline 27 & septembre & $9 \mathrm{~h}$ & Rambouillet & Ile-de-France \\
\hline 28 & septembre & $8 \mathrm{~h}$ & Besançon & Franche-Comté \\
\hline 28 & septembre & $8 \mathrm{~h}$ & Épinal & Lorraine \\
\hline 28 & septembre & $8 \mathrm{~h} 30$ & Nice & Prov.-Alpes-C. d'Azur \\
\hline 28 & septembre & $9 \mathrm{~h}$ & Castres & Midi-Pyrénées \\
\hline 28 & septembre & 9 h 30 & Châteauroux & Centre \\
\hline 28 & septembre & $10 \mathrm{~h}$ & Forest-l'Abbaye & Picardie \\
\hline 29 & septembre & $8 \mathrm{~h} 30$ & Nevers & Bourgogne \\
\hline 29 & septembre & $8 \mathrm{~h} 30$ & Chaumont & Champagne-Ardenne \\
\hline & septembre & $9 \mathrm{~h}$ & Lyons-la-Forêt & Normandie \\
\hline & septembre & 9 h 30 & Boisseuil & Limousin \\
\hline & septembre & appel d'offres & Haute-Savoie & Rhône-Alpes \\
\hline 2 & octobre & 9 h 30 & Aubenas & Rhône-Alpes \\
\hline & octobre & $8 \mathrm{~h}$ & Orléans & Centre \\
\hline & octobre & $9 \mathrm{~h}$ & Aurillac & Auvergne \\
\hline
\end{tabular}


OFFICE NATIONAL DES FORÊTS

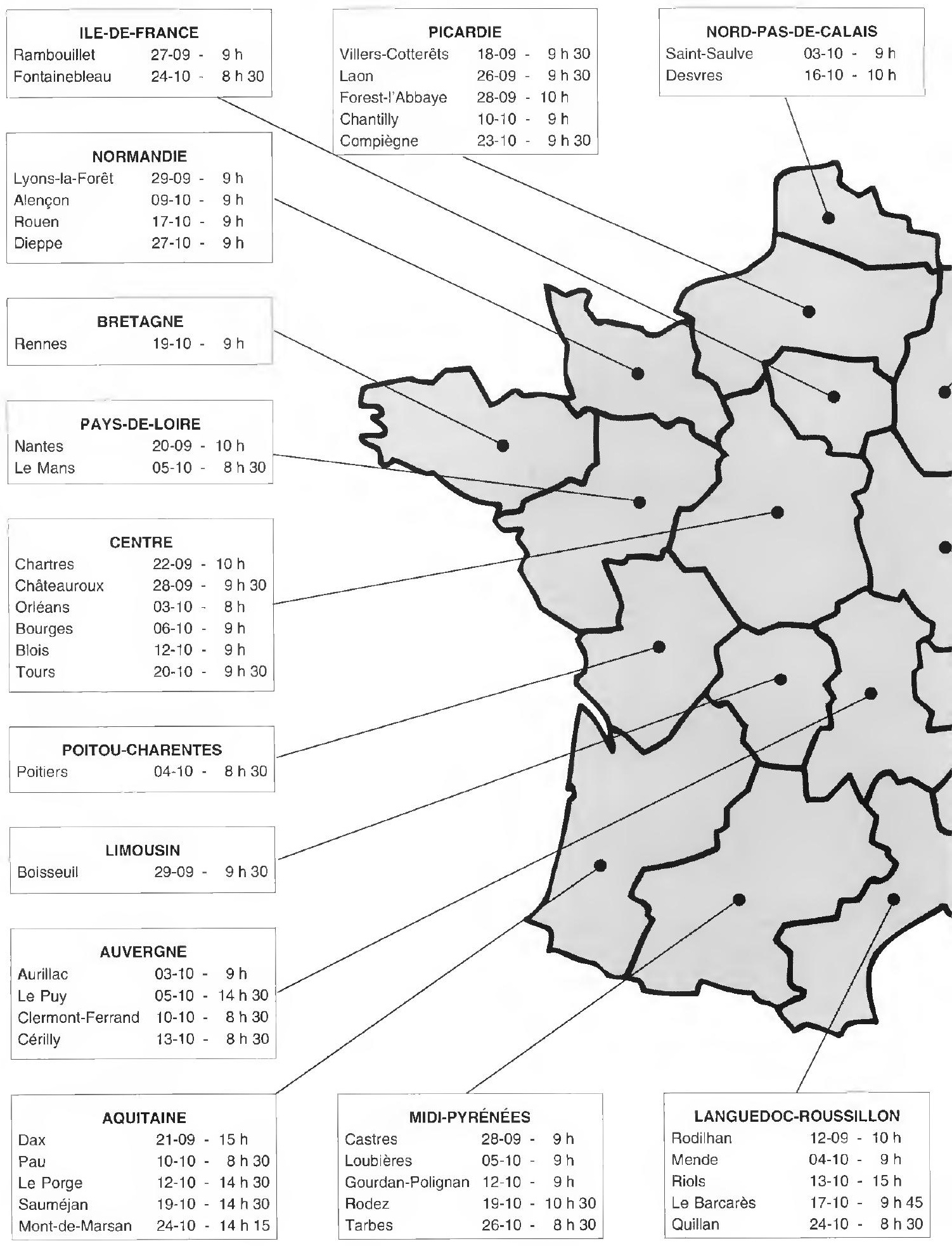




\section{CHAMPAGNE-ARDENNE}

Troyes 13-09 - 8h 30 Saint-Dizier 21-09- $\quad$ h 30 Chaumont 29-09- $8 \mathrm{~h} 30$ Langres $\quad 04-10-8 \mathrm{~h} 30$ Charleville-Mézières 05-10 - 8 h 30 Châlons-sur-Marne 11-10 - 8 h 30
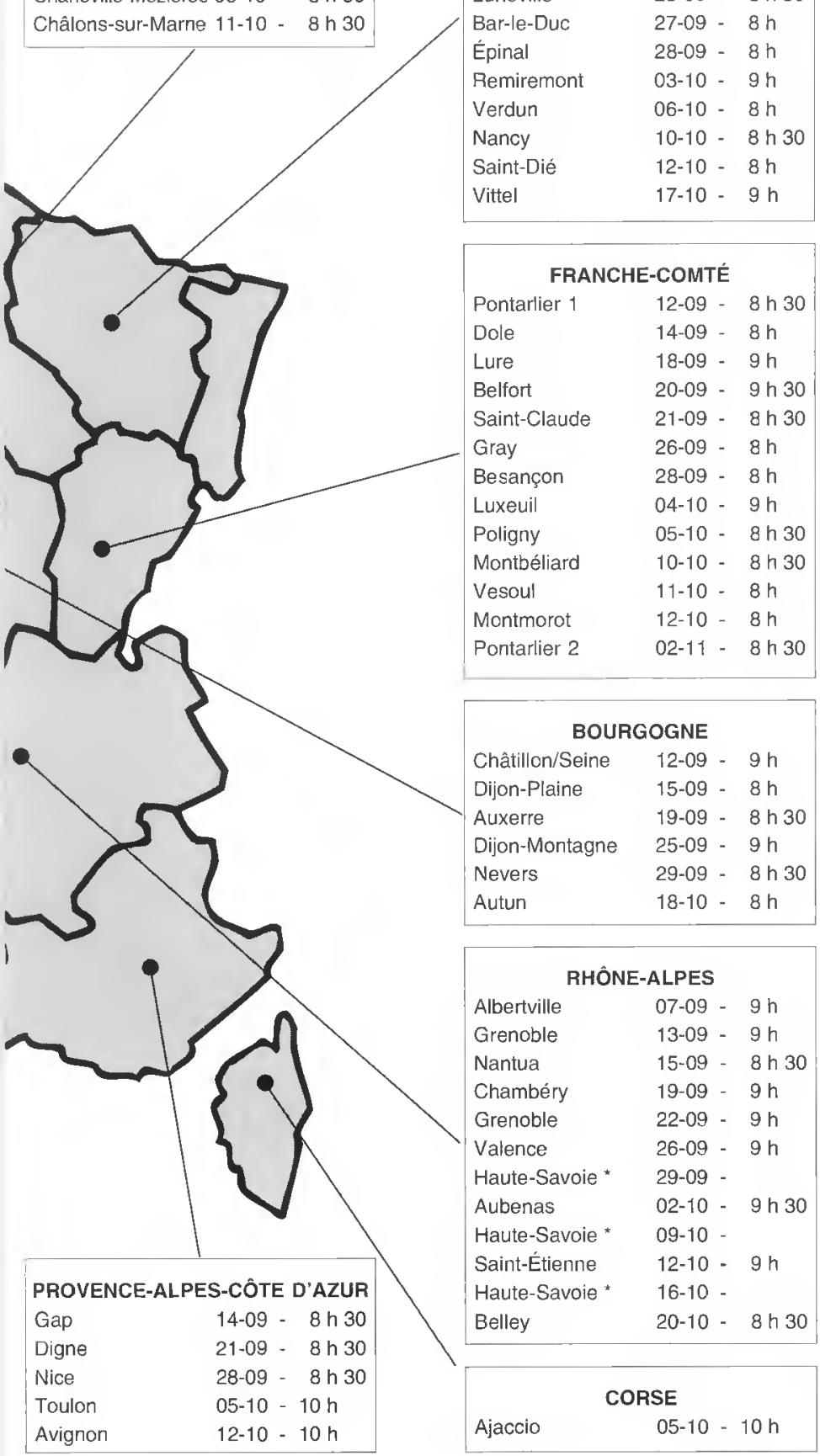

\section{BOURGOGNE}

Châtillon/Seine 12-09 - $9 \mathrm{~h}$

Dijon-Plaine $\quad 15-09-8 \mathrm{~h}$

Auxerre 19-09 - 8h30

Dijon-Montagne 25-09 - $9 \mathrm{~h}$

Nevers 29-09- $8 \mathrm{~h} 30$

Autun

RHÔNE-ALPES

Albertville $07-09-9 \mathrm{~h}$

Grenoble 13-09 - $9 \mathrm{~h}$

Nantua $\quad 15-09-8 \mathrm{~h} 30$

Chambéry 19-09 - $9 \mathrm{~h}$

Grenoble 22-09 - $9 \mathrm{~h}$

Valence 26-09- $9 \mathrm{~h}$

Haute-Savoie * 29-09 -

Aubenas 02-10 - $9 \mathrm{~h} 30$

Haute-Savoie * 09-10 -

Saint-Étienne 12-10 - $9 \mathrm{~h}$

Haute-Savoie * 16-10 -

Belley

$20-10-8$ h 30
GRANDES

VENTES

D'AUTOMNE

1995

LIEUX

DATES

HEURES

DES VENTES

PAR

RÉGIONS 


\begin{tabular}{|c|c|c|c|}
\hline Dates & Heures & Lieux de vente & Régions \\
\hline $\begin{array}{l}3 \text { octobre } \\
3 \text { octobre }\end{array}$ & $\begin{array}{l}9 \mathrm{~h} \\
9 \mathrm{~h}\end{array}$ & $\begin{array}{l}\text { Remiremont } \\
\text { Saint-Saulves }\end{array}$ & $\begin{array}{l}\text { Lorraine } \\
\text { Nord-Pas-de-Calais }\end{array}$ \\
\hline 4 octobre & $8 \mathrm{~h} 30$ & Langres & Champagne-Ardenne \\
\hline 4 octobre & 8 h 30 & Poitiers & Poitou-Charentes \\
\hline 4 octobre & $9 \mathrm{~h}$ & Luxeuil & Franche-Comté \\
\hline 4 octobre & $9 \mathrm{~h}$ & Mende & Languedoc-Roussillon \\
\hline 5 octobre & 8 h 30 & Charleville-Mézières & Champagne-Ardenne \\
\hline 5 octobre & 8 h 30 & Poligny & Franche-Comté \\
\hline 5 octobre & $8 \mathrm{~h} 30$ & Le Mans & Pays-de-Loire \\
\hline 5 octobre & $9 \mathrm{~h}$ & Loubières & Midi-Pyrénées \\
\hline 5 octobre & $10 \mathrm{~h}$ & Ajaccio & Corse \\
\hline 5 octobre & $10 \mathrm{~h}$ & Toulon & Prov.-Alpes-C. d'Azur \\
\hline 5 octobre & 14 h 30 & Le Puy & Auvergne \\
\hline 6 octobre & $8 \mathrm{~h}$ & Verdun & Lorraine \\
\hline 6 octobre & gh & Bourges & Centre \\
\hline 9 octobre & $9 \mathrm{~h}$ & Alençon & Normandie \\
\hline 9 octobre & appel d'offres & Haute-Savoie & Rhône-Alpes \\
\hline 10 octobre & 8 h 30 & $\mathrm{Pau}$ & Aquitaine \\
\hline 10 octobre & 8 h 30 & Clermont-Ferrand & Auvergne \\
\hline 10 octobre & $8 \mathrm{~h} 30$ & Montbéliard & Franche-Comté \\
\hline 10 octobre & 8 h 30 & Nancy & Lorraine \\
\hline 10 octobre & $9 \mathrm{~h}$ & Chantilly & Picardie \\
\hline 11 octobre & $8 \mathrm{~h}$ & Vesoul & Franche-Comté \\
\hline 11 octobre & $8 \mathrm{~h} 30$ & Châlons-sur-Marne & Champagne-Ardenne \\
\hline 12 octobre & $8 \mathrm{~h}$ & Montmorot & Franche-Comté \\
\hline 12 octobre & $8 \mathrm{~h}$ & Saint-Dié & Lorraine \\
\hline 12 octobre & $9 \mathrm{~h}$ & Blois & Centre \\
\hline 12 octobre & $9 \mathrm{~h}$ & Gourdan-Polignan & Midi-Pyrénées \\
\hline 12 octobre & $9 \mathrm{~h}$ & Saint-Étienne & Rhône-Alpes \\
\hline 12 octobre & $10 \mathrm{~h}$ & Avignon & Prov.-Alpes-C. d'Azur \\
\hline 12 octobre & $14 \mathrm{~h} 30$ & Le Porge & Aquitaine \\
\hline 13 octobre & $8 \cap 30$ & Cerilly & Auvergne \\
\hline 13 octobre & $15 \mathrm{~h}$ & Riols & Languedoc-Roussillon \\
\hline 16 octobre & $10 \mathrm{~h}$ & Desvres & Nord-Pas-de-Calais \\
\hline 16 octobre & appel d'offres & Haute-Savoie & Rhône-Alpes \\
\hline 17 octobre & $9 \mathrm{~h}$ & Vittel & Lorraine \\
\hline 17 octobre & $9 \mathrm{~h}$ & Rouen & Normandie \\
\hline 17 octobre & 9 h 45 & Le Barcarès & Languedoc-Roussillon \\
\hline 18 octobre & $8 \mathrm{~h}$ & Autun & Bourgogne \\
\hline 19 octobre & $9 \mathrm{~h}$ & Rennes & Bretagne \\
\hline 19 octobre & 10 h 30 & Rodez & Midi-Pyrénées \\
\hline 19 octobre & $14 \mathrm{~h} 30$ & Sauméjan & Aquitaine \\
\hline 20 octobre & $8 \mathrm{~h} 30$ & Belley & Rhône-Alpes \\
\hline 20 octobre & 9 h 30 & Tours & Centre \\
\hline 23 octobre & $9 \mathrm{~h} 30$ & Compiègne & Picardie \\
\hline 24 octobre & $8 \mathrm{~h} 30$ & Fontainebleau & lle-de-France \\
\hline 24 octobre & $8 \mathrm{~h} 30$ & Quillan & Languedoc-Roussillon \\
\hline 24 octobre & $14 \mathrm{~h} 15$ & Mont-de-Marsan & Aquitaine \\
\hline 26 octobre & $8 \mathrm{~h} 30$ & Tarbes & Midi-Pyrénées \\
\hline 27 octobre & $9 \mathrm{~h}$ & Dieppe & Normandie \\
\hline 2 novembre & $8 \mathrm{~h} 30$ & Pontarlier 2 & Franche-Comté \\
\hline
\end{tabular}

